\title{
Desenvolvimento dos estágios imaturos de Palmistichus elaeisis Delvare \& LaSalle (Hymenoptera, Eulophidae) em pupas de Lepidoptera
}

\author{
Maria A. L. Bittencourt ${ }^{1} \&$ Evoneo Berti Filho ${ }^{2}$
}

\begin{abstract}
${ }^{1}$ Departamento de Ciências Agrárias e Ambientais, Universidade Estadual de Santa Cruz. Rodovia Ilhéus-Itabuna, Km 16, $45650-000$ Ilhéus-BA, Brasil. Endereço eletrônico: malbitte@uesc.br

${ }^{2}$ Departamento de Entomologia, Fitopatologia e Zoologia Agrícola, Escola Superior de Agricultura "Luiz de Queiroz," Universidade de São Paulo. Caixa Postal 9, 13418-900 Piracicaba-SP, Brasil. Endereço eletrônico: eberti@carpa.ciagri.usp.br
\end{abstract}

\begin{abstract}
Aвstract. Development of immature stages of Palmistichus elaeisis Delvare \& LaSalle (Hymenoptera, Eulophidae) in Lepidoptera pupae. Palmistichus elaeisis Delvare \& LaSalle, 1993 is an endoparasitoid collected from pupae of Sabulodes sp. (Lepidoptera, Geometridae). The immature stages of this parasitoid were studied in laboratory $\left(25 \pm 1{ }^{\circ} \mathrm{C} ; 70 \pm 10 \%\right.$ RH; 14-hour photoperiod) in the following Lepidoptera pupae: Diatraea saccharalis (Fabricius, 1794) (Crambidae), Anticarsia gemmatalis Hübner, 1818, Heliothis virescens (Fabricius, 1781), Spodoptera frugiperda (J. E. Smith, 1797) (Noctuidae) and Thyrinteina arnobia (Stoll, 1782) (Geometridae). It was observed that the eggs and the $1^{\text {st }}$ instar larvae are hyaline and hymenopteriform; $2^{\text {nd }}, 3^{\text {rd }}$ and $4^{\text {th }}$ instars larvae are whitish and 12 -segmented. The host species did not influence the number of instars.
\end{abstract}

KEYWORDS. Biology; Eulophidae; parasitoid.

Resumo. Palmistichus elaeisis Delvare \& LaSalle, 1993 é um endoparasitóide coletado de pupas de Sabulodes sp. (Lepidoptera, Geometridae). Os estágios imaturos deste parasitóide foram estudados em laboratório $\left(25 \pm 1{ }^{\circ} \mathrm{C} ; 70 \pm 10 \%\right.$ UR; fotofase 14 h) em pupas dos seguintes lepidópteros: Diatraea saccharalis (Fabricius, 1794) (Crambidae), Anticarsia gemmatalis Hübner, 1818, Heliothis virescens (Fabricius, 1781), Spodoptera frugiperda (J. E. Smith, 1797) (Noctuidae) e Thyrinteina arnobia (Stoll, 1782) (Geometridae). Observou-se que os ovos e as larvas de $1^{\circ}$ ínstar são hialinas e himenopteriformes; as larvas dos $2^{\circ}, 3^{\circ}$ e $4^{\circ}$ ínstares são esbranquiçadas e 12 segmentadas. A espécie de hospedeiro não influenciou o número de ínstares.

Palavras-Chave. Biologia; Eulophidae; parasitóide.

Os himenópteros parasitóides são aqueles cujas larvas se desenvolvem em outro artrópode, usualmente um inseto, acarretando a sua morte ao final do seu desenvolvimento. Os eulofídeos predominam como parasitóides de estágios imaturos de lepidópteros, coleópteros, dípteros e himenópteros, especialmente daqueles abrigados em tecidos de plantas, como as larvas minadoras, os formadores de galhas e as brocas de tronco. As espécies de Tetrastichinae (Eulophidae) possuem uma grande variedade de hospedeiros (HANSON \& GAULD 1995). Palmistichus elaeisis Delvare \& LaSalle, 1993 foi registrada no Brasil parasitando Eupseudosoma involuta (Sepp, 1852) (Lepidoptera, Arctiidae) e Euselasia eucerus Hewitson, 1872 (Lepidoptera, Riodinidae) em eucalipto (Delvare \& LaSalle, 1993).

Na maioria dos himenópteros parasitóides os ovos são do tipo himenopteriforme, de coloração clara; as larvas são fusiformes, ápodes, com tegumento liso, de coloração clara ou translúcida, podendo apresentar pequenos espinhos ou cerdas; a cabeça é normalmente hipognata; possuem de 12 a 13 segmentos; os espiráculos freqüientemente estão presentes no mesotórax ou metatórax; são sedentárias e apresentam mesêntero cego e geralmente passam por três a cinco ínstares. Podem apresentar o estágio de pré-pupa, com o estágio de pupa ocorrendo em variadas situações (CLAUSEN 1940; HAGEN 1964; DoutT et al. 1976; GAuld \& Bolton 1988; Hanson \&
Gauld 1995; LaSalle \& Schauff 1995). A determinação do número de ínstares é considerada importante no estudo da biologia dos insetos. A regra de Dyar se baseia na medida da cápsula cefálica e é amplamente usada para lepidópteros, sendo também validada para outras ordens, inclusive Hymenoptera (PARRA \& HADDAD 1989). Em casos onde a regra de Dyar não se aplica, a determinação do número de ínstares e do tamanho médio das cápsulas cefálicas de cada ínstar, pode ser feita através do modelo de regressão segmentado (Ambrosano et al. 1997). Ohashi (1984) estudou, em laboratório, o desenvolvimento e aspectos morfológicos do calcidídeo Brachymeria ovata (Say, 1824) sobre pupas de Spodoptera frugiperda (J. E. Smith, 1797) (Lepidoptera, Noctuidae), utilizando como parâmetros para determinação do número de ínstares o diâmetro do espiráculo, o comprimento da barra transversal do tentório, a largura da máscara bucal e a largura da cápsula cefálica.

Vários trabalhos têm sido realizados sobre o desenvolvimento de himenópteros parasitóides de pupas em espécies de importância agrícola ou florestal, embora sobre eulofídeos as pesquisas sejam escassas. BlEDSOE et al. (1983) estudaram as fases do desenvolvimento do eulofídeo Pediobius foveolatus (Crawford, 1912), parasito larval de Epilachna varivestis Mulsant, 1850 (Coleoptera, Coccinellidae). Román (1996) estudou, em laboratório, os 


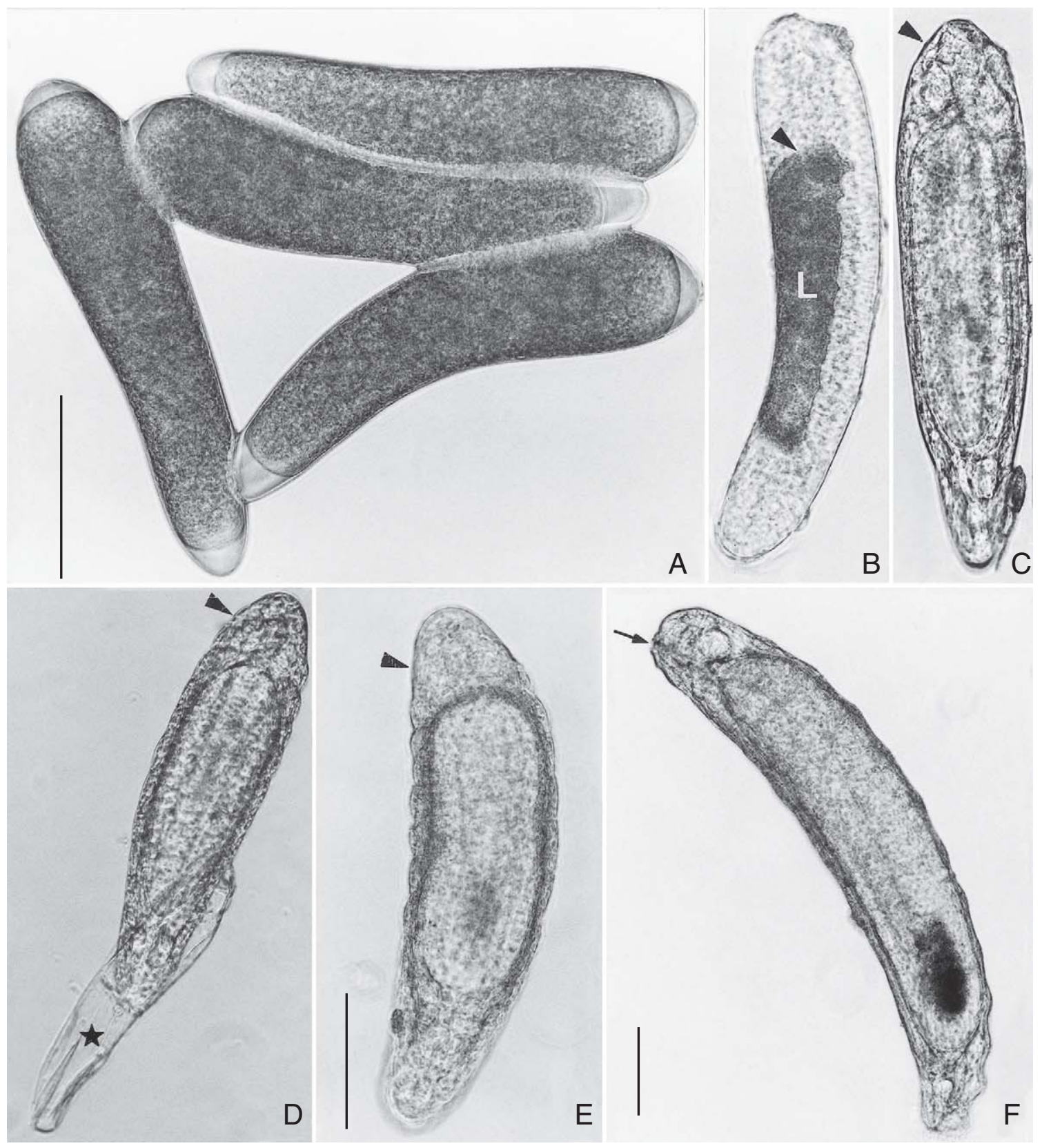

Fig. 1. Desenvolvimento embrionário de Palmistichus elaeisis. A, ovos recém-colocados, 260x; B, ovo com larva em desenvolvimento (L), 260x; C, ovo com larva completamente desenvolvida, 260x.; D, larva eclodida (48 h); E, larva recém-eclodida, 210x; F, larva típica do 1ํínstar $(72 \mathrm{~h})$. As pontas de seta indicam a região anterior das larvas; $(\star)=$ córion, 240x; $(\rightarrow)=$ mandíbula, 120x. Escala=0,1 mm.

aspectos biológicos e a morfologia dos estágios imaturos do eulofídeo Tetrastichus sp., parasitóide de pupas de Methona confusa psamathe Godman \& Salvin, 1898 (Lepidoptera, Ithomiidae); além do hospedeiro natural, utilizou como hospedeiros alternativos Leptophobia aripa (Boisduval,1836) e Colias lesbia (Fabricius, 1775) (Lepidoptera, Pieridae) com excelentes resultados.

Este trabalho teve como objetivos caracterizar morfologicamente as fases imaturas de P. elaeisis e avaliar seu desenvolvimento sobre pupas de cinco espécies de lepidópteros criados com dieta artificial.

\section{MATERIAL E MÉTODOS}

A pesquisa foi desenvolvida no Laboratório de Controle Biológico do Departamento de Entomologia, Fitopatologia e Zoologia Agrícola da Escola Superior de Agricultura "Luiz de Queiroz", da Universidade de São Paulo, em Piracicaba, São Paulo. O parasitóide P. elaeisis, obtido de pupas de Sabulodes sp. (Lepidoptera, Geometridae) coletadas em área de eucalipto, foi mantido em gaiola de madeira $(70 \times 50 \times 40 \mathrm{~cm})$ com vidro na parte frontal, a $25 \pm 1^{\circ} \mathrm{C}, 70 \pm 10 \%$ de umidade relativa e $14 \mathrm{~h} \mathrm{de}$ fotofase. Os adultos foram alimentados com solução de mel a 


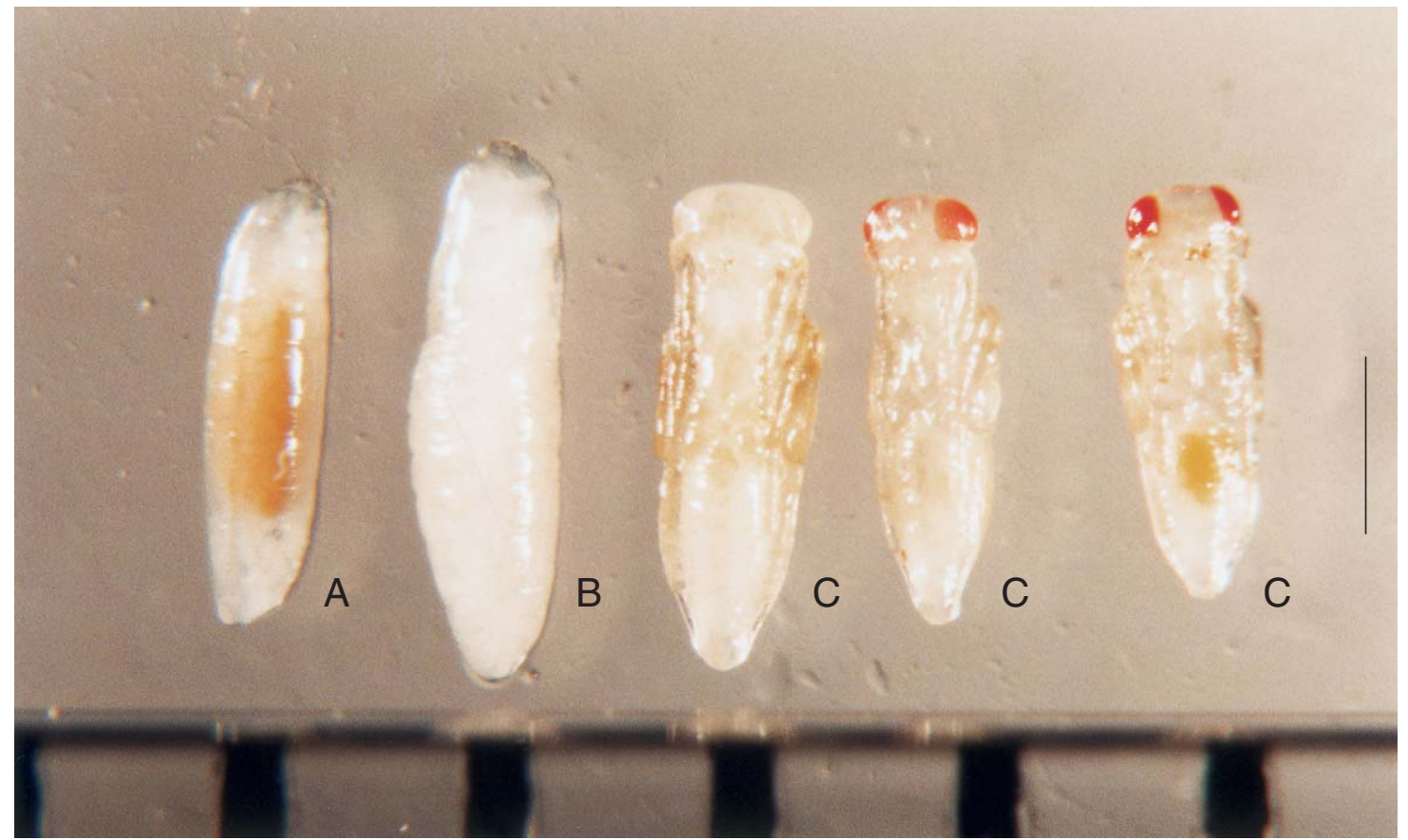

Fig. 2. Formas imaturas de Palmistichus elaeisis. A, larva; B, pré-pupa; C, pupas. Escala=0,1 mm.

$10 \%$ e pupas das seguintes espécies de lepidópteros, Diatraea saccharalis (Fabricius, 1794) (Crambidae), Anticarsia gemmatalis Hübner, 1818, Heliothis virescens (Fabricius, 1781), Spodoptera frugiperda (Noctuidae) e Thyrinteina arnobia (Stoll, 1782) (Geometridae), foram utilizadas para a manutenção da criação. Pupas hospedeiras, com 24 a 72 horas de idade, obtidas de criação com dieta artificial, foram expostas por $24 \mathrm{~h}$ aos parasitóides na gaiola; após este período, foram individualizadas e deixadas em câmaras climatizadas, a $25 \pm$ $1^{\circ} \mathrm{C}, 70 \pm 10 \%$ de UR e fotofase de 14 horas. Durante 16 dias, foram retiradas diariamente de três a cinco pupas de cada hospedeiro e armazenadas em um "freezer" para observações posteriores sobre a fase de desenvolvimento do parasitóide. A duração de cada estágio de desenvolvimento foi avaliada em 20 pupas de cada hospedeiro, totalizando 100 pupas. Após a dissecação das pupas sob microscópio estereoscópio, alguns parasitóides de cada hospedeiro foram lavados em solução salina $(0,9 \%)$, para possibilitar a medição do comprimento e largura do corpo e a largura da cápsula cefálica de 20 formas imaturas, com o auxílio de uma ocular graduada e observar diferentes características. Em seguida, o material foi colocado em tubos plásticos Eppendorf etiquetados, com solução fixadora padrão ( $2 \%$ de glutaraldeido, $2 \%$ de paraformaldeido, 0,05M tampão cacodilato, 0,001 $\mathrm{M} \mathrm{C}_{\mathrm{a}} \mathrm{Cl}_{2} \mathrm{e}$ água destilada) e mantidos em geladeira até serem fotografados. Alguns dos exemplares medidos foram preparados e fotografados: os ovos em fotomicroscópio, equipado com contraste de fase no Laboratório de Biologia Celular da Universidade Estadual de Campinas, São Paulo, para observação do desenvolvimento embrionário; as larvas e pupas em microscópio estereoscópio no Laboratório de
Biologia (ESALQ/USP). O material encontra-se depositado no Departamento de Entomologia, Fitopatologia e Zoologia Agrícola da Escola Superior de Agricultura "Luiz de Queiroz".

\section{RESULTADOS}

Os ovos são hialinos, lisos, tipicamente himenopteriforme e apresentam a região anterior mais estreita. O período de incubação durou, aproximadamente, 48 horas nos diferentes hospedeiros (Fig. 1). As larvas do 1º́nstar são ápodes com a cutícula transparente, formato himenopteriforme com 12 segmentos; as larvas dos 2oㅡ $3^{\circ}$ e 4o ínstares (5 a 10 dias)

Tabela I. Dimensões em mm (média \pm erro padrão), das diferentes fases de desenvolvimento de Palmistichus elaeisis em pupas de lepidópteros. Temperatura $25 \pm 1^{\circ} \mathrm{C}$; UR $70 \pm 10 \%$; fotofase $14 \mathrm{~h}$.

\begin{tabular}{lccc}
\hline $\begin{array}{l}\text { Ontogenia } \\
(\mathrm{n}=200)\end{array}$ & $\begin{array}{c}\text { Comprimento } \\
\text { do corpo }\end{array}$ & $\begin{array}{c}\text { Largura } \\
\text { do corpo }\end{array}$ & $\begin{array}{c}\text { Largura da } \\
\text { cápsula cefálica }\end{array}$ \\
\hline Ovo & $0,30 \pm 0,003$ & 0,05 &.. \\
Larva I & $0,71 \pm 0,02$ & $0,18 \pm 0,006$ & $0,07 \pm 0,003$ \\
Larva II & $2,06 \pm 0,03$ & $0,42 \pm 0,005$ & $0,11 \pm 0,002$ \\
Larva III & $2,55 \pm 0,03$ & $0,56 \pm 0,006$ & $0,18 \pm 0,002$ \\
Larva IV & $2,51 \pm 0,04$ & $0,57 \pm 0,006$ & $0,20 \pm 0,002$ \\
& & & \\
Pré-pupa & $2,00 \pm 0,03$ &.. & $0,18 \pm 0,002$ \\
Pupa & $1,98 \pm 0,02$ &.. & $0,55 \pm 0,005$ \\
Pupa fêmea & $2,09 \pm 0,02$ &.. & $0,58 \pm 0,005$ \\
Pupa macho & $1,61 \pm 0,01$ &.. & $0,44 \pm 0,003$ \\
\hline
\end{tabular}




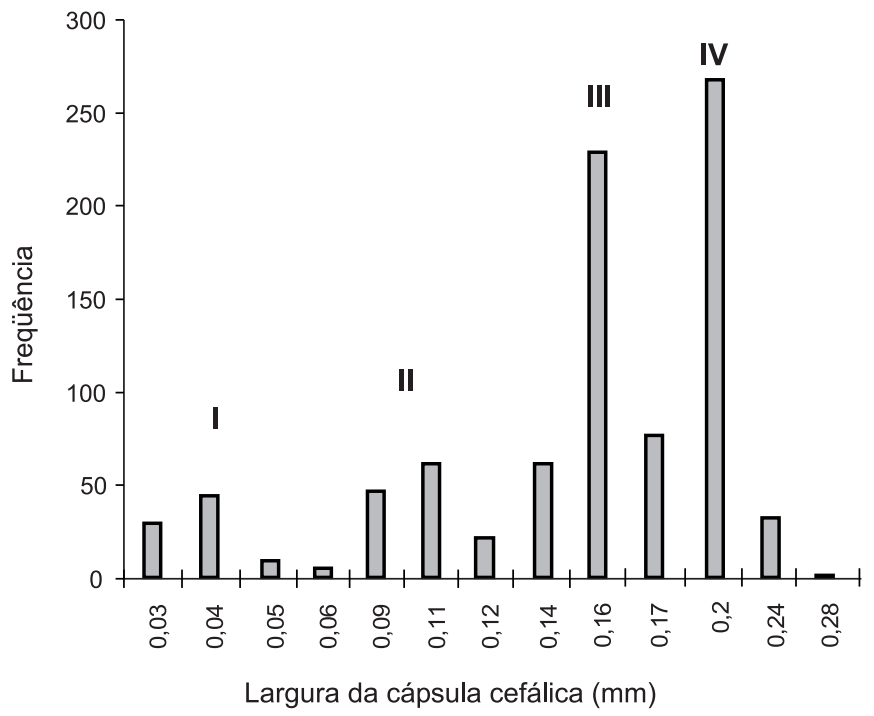

Fig. 3. Distribuição de freqüências de medidas da largura da cápsula cefálica dos ínstares larvais (I a IV) do parasitóide Palmistichus elaeisis, nos diferentes hospedeiros de Lepidoptera.

possuem coloração esbranquiçada e segmentação bem definida (Fig. 2). O estágio larval durou 8,04 $\pm 0,17$ dias nos hospedeiros utilizados.

A pré-pupa é de cor branca e com apêndices pigmentados (Fig. 2). O estágio de pré-pupa foi de aproximadamente 24 horas, em todos os hospedeiros testados, e observou-se a eliminação do mecônio após o último ínstar larval. O período pupal foi de 9,8 $\pm 0,17$ dias. Até a diferenciação sexual, a coloração das pupas é esbranquiçada e com olhos vermelhos. As pupas de fêmeas são, geralmente, maiores em comprimento do que as dos machos e a partir do $14^{\circ}$ dia de desenvolvimento, quando ocorre o escurecimento do tegumento e dos olhos, observa-se a diferenciação entre fêmeas (escurecimento do ovipositor) e machos (placa ventral no escapo). Foram registradas as dimensões das fases de desenvolvimento de $P$. elaeisis nos diferentes hospedeiros testados (Tabela I). O desenvolvimento pós-embrionário apresenta quatro ínstares larvais; o número de ínstares foi determinado pela distribuição de freqüências das medidas de cápsula cefálica das fases larvais de P. elaeisis (Fig. 3, Tabela I), sendo o coeficiente de determinação de $99 \%$ e constante de Dyar (K), 1,40. Verificouse que a espécie de hospedeiro (Lepidoptera) não influencia o número de ínstares.

Ocorreu o desenvolvimento do parasitóide nos cinco hospedeiros testados, verificando-se a possibilidade destes hospedeiros serem utilizados em criação massal, corroborando os dados obtidos por Bittencourt \& Berti Filho (1999) que observaram a não preferência de $P$. elaeisis para oviposição nos hospedeiros A. gemmatalis, D. saccharalis, $H$. virescens, S. frugiperda e T. arnobia.

Agradecimentos. Os autores agradecem ao Dr. Marcelo Teixeira Tavares da Universidade Federal do Espírito Santo, Brasil e Dr. John LaSalle, CSIRO, Austrália, pela identificação do parasitóide.

\section{REFERÊNCIAS}

Ambrosano, G. M. B.; T. Igue \& A. L. LourençÃo. 1997. Determinação do número de instares de insetos utilizando modelo de regressão segmentado. Anais da Sociedade Entomológica do Brasil 26(2): 371-374.

Bittencourt, M. A. L. \& E. Berti Filho. 1999. Preferência de Palmistichus elaeisis por pupas de diferentes lepidópteros praga. Scientia Agricola 56(4): 1281-1283.

Bledsoe, L. W.; R. V. Flanders \& C. R. Edwards. 1983. Morphology and development of the immature stages of Pediobius foveolatus (Hymenoptera: Eulophidae). Annals of the Entomological Society of America 76(6): 953-957.

Clausen, C. P. 1940. Entomophagous Insects. New York, McGrawHill Book Co, $\mathrm{x}+688$ p.

Delvare, G. \& J. Lasalle. 1993. A new genus of Tetrastichinae (Hymenoptera: Eulophidae) from the Neotropical Region, with the description of a new species parasitic on key pests of oil palm. Journal of Natural History 27: 435-444.

Doutt, R. L.; D. P. Annecke \& E. Tremblay. 1976. Biology and host relationships of parasitoids, p.143-168. In: C. B. Huffaker \& P. S. Messenger (Eds.) Theory and practice of biological control. New York, Academic Press, xxi+788 p.

Gauld, I. \& B. Bolton. (Eds.) 1988. The Hymenoptera. New York, Oxford University Press, xi+332 p.

Hagen, K. S. 1964. Developmental stages of parasites, p. 168-246. In: P. DЕвАсн (Ed.). Biological control of insect pests and weeds. New York, Reinhold Publishing Corporation, xxiv+844 p.

Hanson, P. E. \& I. D. Gauld. 1995. The biology of hymenoptera. Natural history, p. 20-28. In: P. E. Hanson \& I. D. GAuld (Eds.). The Hymenoptera of Costa Rica. New York, Oxford University Press, 893 p.

Lasalle, J. \& M. E. Schauff. 1995. The chalcidoid families. Eulophidae, p. 315-329. In: P. E. Hanson, \& I. D. Gauld (Eds.). The Hymenoptera of Costa Rica. New York, Oxford University Press, $893 \mathrm{p}$.

Оназні, O. S. 1984. Biologia e aspectos morfológicos de Brachymeria ovata (Say, 1824) (Hymenoptera: Chalcididae) endoparasito de pupas de Lepidoptera. Tese de Doutorado. Piracicaba, Escola Superior de Agricultura "Luiz de Queiroz", Universidade de São Paulo, 90 p.

Parra, J. R. P. \& M. L. Hadad. 1989. Determinação do número de ínstares de insetos. Piracicaba, FEALQ, 49 p.

Román, L. E. N. 1996. Morphology of the immature stages and biological aspects of Tetrastichus sp. (Hymenoptera: Tetrastichinae), parasitoid of Methona confusa psamathe Godm. et Salv. (Lepidoptera: Ithomiidae). Neotropica 42: 41-46. 